\title{
Conjunctival biopsy in the diagnosis of sarcoidosis
}

\author{
Richard F Spaide, David L Ward
}

\begin{abstract}
We prospectively studied 47 sarcoidosis suspects and compared conjunctival and transbronchial lung biopsies in these patients. Thirty-four patients had positive findings on biopsy by either method. The transbronchial biopsy was positive in 31 patients, and the conjunctival was positive in 19 . The transbronchial lung biopsy was more likely to be positive in black patients $(p=0.009)$ and in patients with pulmonary infiltrates on chest $x$ ray $(p=0 \cdot 0044)$. In comparison, the conjunctival biopsy was more likely to be positive in patients with conjunctival follicles $(p=0.036)$, ocular abnormalities consistent with sarcoidosis $(p=0.02)$, and in patients with pulmonary infiltrates on chest $x$ ray $(p=0.029)$. Iritis was present in 12 patients, enlarged lacrimal glands in three, and vitritis in five. We conclude that the conjunctival biopsy is an effective means of diagnosing sarcoidosis and that every sarcoidosis patient should have an ophthalmic examination.
\end{abstract}

Sarcoidosis is a granulomatous disorder of multiple organ systems that has no known cause, has an unpredictable course, and can mimic many other diseases. It is predominantly found in young adults, who frequently have bilateral hilar adenopathy, pulmonary infiltration, respiratory problems, skin lesions, and ocular problems. ${ }^{1-7}$ The diagnosis of sarcoidosis is best supported when histological evidence supports the clinical and radiological findings. ${ }^{\prime}$

The granulomas are usually widely distributed, so the biopsy site should be determined by the risks versus the likelihood of success. Transbronchial lung biopsy (TBBX) by a flexible fibreoptic bronchoscope is currently the biopsy procedure of choice in making the diagnosis of sarcoidosis. ${ }^{89}$ However, conjunctival biopsy (CBX) has frequently been reported to be a simple procedure that is helpful. ${ }^{10-17} \mathrm{We}$ conducted a prospective study of transbronchial and conjunctival biopsies in 47 patients suspected of having sarcoidosis on clinical grounds, and compared the rates of positive biopsy results for the two procedures. We also catalogued the ocular findings to determine the incidence of ocular abnormalities in newly diagnosed sarcoidosis patients.

\section{Patients and methods}

This study was performed at a medical centre serving both local and referred patients. We considered any patient who had symptoms or findings suggesting a differential diagnosis that included sarcoidosis to be a sarcoidosis suspect. We used the definition of sarcoidosis from the Seventh International Conference on Sarcoidosis ${ }^{1}$ as our case definition. During the study period 49 consecutive patients met the entrance criteria of sarcoidosis as a differential diagnosis. The nature and purpose of the biopsy procedures were explained to the patients, who gave written informed consent. Two patients chose not to participate in the study; this report is on the remaining 47.

All patients had skin testing with intermediate purified protein derivative of tuberculin (PPD) and an anergy panel, and none of these patients had a positive reaction to the tuberculin antigen. Each patient had chest $x$ rays, and these $x$ rays were graded into four stages according to conventional methods. ${ }^{18}$ Stage 0 is normal, and stage 1 has bilateral hilar adenopathy. Stage 2 has enlarged hilar nodes with pulmonary opacities, and stage 3 has pulmonary infiltrates without node enlargement. Every patient had function tests (PFT). For our analysis we examined the forced vital capacity, forced expiratory volume in 1 second, forced expiratory flow, $25-75 \%$ maximum voluntary ventilation, and total lung capacity. Each subtest was considered abnormal if it was less than $75 \%$ of the predicted value for patients the same age, sex and size.

Each sarcoidosis suspect was examined by a chest specialist, who obtained at least six and usually eight to 10 random transbronchial biopsies from each patient with a fibreoptic bronchoscope. We obtained angiotensin converting enzyme (ACE) levels in 29 patients. Every patient had a complete ocular examination by an ophthalmologist. A strip of conjunctiva 10 to $12 \mathrm{~mm}$ long was excised from each eye, and any conjunctival abnormality noted on slit-lamp examination was included in the biopsy when possible. The biopsy specimens were fixed in formalin and embedded in paraffin blocks, were sectioned (Fig 1), and stained with haematoxylin and eosin. If a biopsy showed non-caseating granulomas, it was stained for fungal and acid fast organisms. If no organisms were present, the biopsy was considered to be positive for sarcoidosis (Fig 2).

History, physical and ocular findings, PFT results, ACE levels, chest $x$ ray stage, and biopsy findings of the 47 patients were entered on to a form adapted for use with a computer database. The data were evaluated with the help of descriptive statistics and, for frequency data, Fisher's exact test of probability. For analysis the chest $x$ ray staging was divided into two groups. Stages 0 and 1 chest $x$ rays were grouped together and called pulmonary infiltrate negative; stages 2 and 3 were group together and called infiltrate positive. We classified chest $x$ ray results in this manner because our sample size was small and also chest $x$ ray stages in sarcoidosis are not strictly ordinal, ${ }^{18}$ so that making four strata for the $x$ rays violates the assumptions in the statistical methods used. We rejected the null 


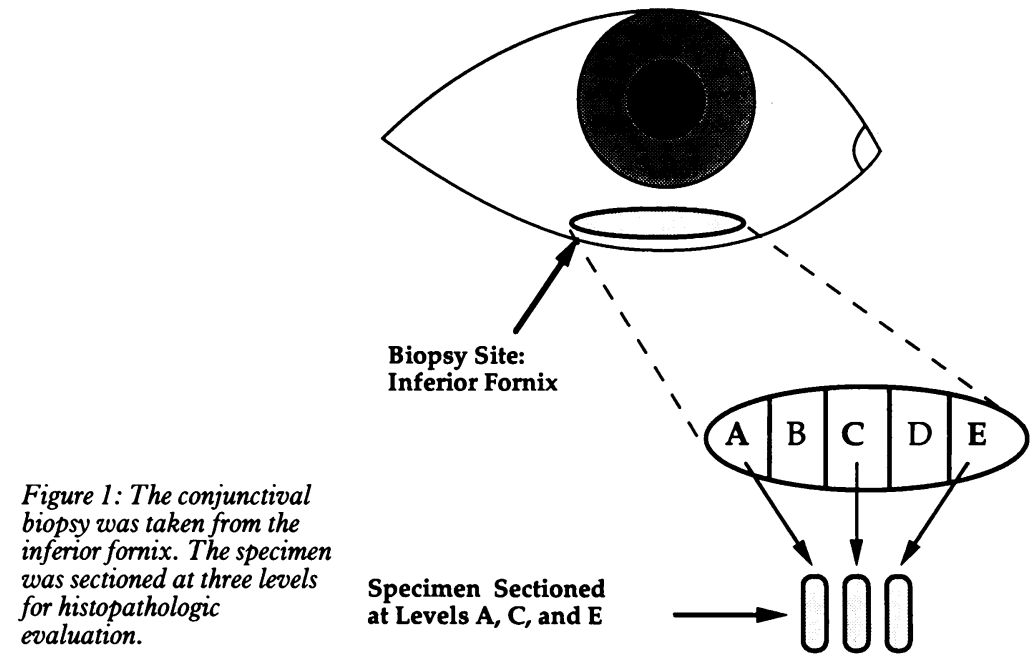

hypothesis if the probability of committing a type I error was 0.05 or less.

\section{Results}

Of the 47 patients studied 31 were male (24 blacks, 7 Caucasians) and 16 female ( 11 blacks, 5 Caucasians). The mean age was $29 \cdot 4$ years (SD $6 \cdot 2$ years), while the average duration of symptoms was 6.2 months (SD $9 \cdot 1$ months). There was no statistically significant difference in age or duration of symptoms among males and females or Caucasians and blacks.

Conjunctival follicles were found in 12 patients. Three patients had enlarged lacrimal glands. Iritis was present in 12 patients $(25 \cdot 5 \%)$, keratic precipitates were present in $4(8.5 \%)$. Five patients $(10.6 \%)$ had anterior vitritis, with four of these having snowballs in the vitreous. All patients with intraocular inflammation were black $(\mathrm{p}=0.016)$. Any ocular abnormality consistent with sarcoidosis - that is, conjunctival follicles, enlarged lacrimal glands, or intraocular

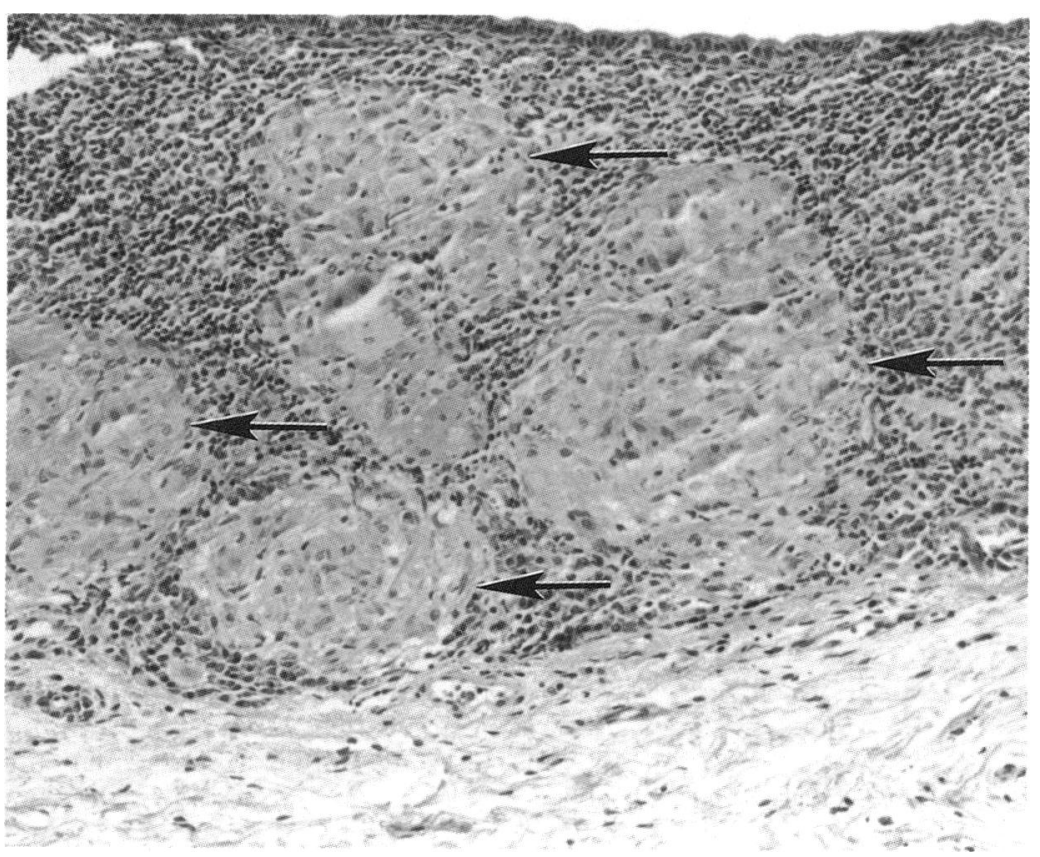

Figure 2: Photomicrograph of a conjunctival biopsy with arrows showing individual noncaseating granulomas. (Haematoxylin and eosin $\times 120$.) inflammation - was found in 20 patients, and 18 were black.

Of the 47 patients $31(66 \%)$ had a positive transbronchial biopsy and $19(40.4 \%)$ had a positive conjunctival biopsy. A total of 34 were discovered to have sarcoidosis by one or both of these two biopsy methods. Of the 13 patients who did not have a positive TBBX or CBX one had a lymph node biopsy positive for sarcoidosis; four patients had additional lung biopsies, one of which was positive; one patient with asymmetrical hilar adenopathy had two non-diagnostic TBBX procedures, and he was discovered to have sarcoidosis by mediastinoscopy; one patient was lost to follow-up; the remaining patients, including one who had a stable chest $x$ ray for seven years, were followed up without any additional biopsies. Thirty-seven patients were eventually found to have histological evidence of sarcoidosis of whom conjunctival biopsy discovered $51 \%$ and transbronchial lung biopsy $84 \%$.

Transbronchial biopsies were positive in $77 \cdot 1 \%$ and conjunctival biopsies in $45 \cdot 7 \%$ of black sarcoidosis suspects. Caucasian sarcoidosis suspects had $33.3 \%$ and $25 \%$ positive TBBX and $\mathrm{CBX}$ respectively. The difference between the two races was significant for the TBBX ( $p=$ 0.009 ) but not for the conjunctival biopsy ( $p=$ $0 \cdot 18$ ). Patients with pulmonary infiltrates had a positive TBBX $80 \cdot 6 \%$ of the time, while $37 \cdot 5 \%$ of patients with no pulmonary infiltrates had a positive biopsy $(p=0 \cdot 0044)$. Likewise, the conjunctival biopsy was more likely to be positive in patients with pulmonary infiltrates than patients without infiltrates, $51 \cdot 6 \%$ versus $23 \cdot 1 \%(\mathrm{p}=$ $0 \cdot 029$ ). Conjunctival biopsies were positive more often in sarcoidosis suspects with follicles, $66 \cdot 7 \%$, than those with a normal conjunctiva $31 \cdot 4 \%(\mathrm{p}=$ 0.036 ). Conjunctival biopsies were positive in $60 \%$ of those with any eye abnormality consistent with sarcoidosis compared with $25.9 \%$ of those with no abnormalities on ocular examination $(p=0.02)$. We could find no element of the PFT, singly or in combination, that correlated with a positive TBBX or CBX.

The diagnosis of sarcoidosis was established in 10 of the 12 suspects with conjunctival follicles, and the conjunctival biopsy detected $8(80 \%)$ of these. Any ocular abnormality consistent with sarcoidosis was found in 20 , and 18 were eventually found to have sarcoidosis. The conjunctival biopsy detected 12 of these $(66 \%)$.

The ACE test was abnormal in $62 \%$ of those with positive biopsies. Black patients, patients with intraocular inflammation, or patients with more advanced chest $x$ ray stages were not more likely to have an abnormal ACE test.

\section{Discussion}

More than one-half of the sarcoidosis patients $(54 \cdot 1 \%)$ had some type of ocular abnormality, and $32.4 \%$ had intraocular inflammation. These values are greater than in previous studies being $34 \%$ and $28 \%$ respectively. The previous investigations were retrospective studies from sarcoidosis centres, and it was difficult to determine if the patients were being treated for sarcoidosis at the time of their ocular examination. We 
performed a prospective study of untreated patients. All our patients were examined by an ophthalmologist in contrast to some of the studies where only selected patients were examined by an ophthalmologist. Because of our findings we would strongly recommend early ophthalmic consultation for every sarcoidosis patient.

The conjunctival biopsy was positive in $40.4 \%$ of suspects. Again, this number is hard to compare with previous reports. Past studies frequently included patients with known sarcoidosis, included biopsies only in patients with $^{13}$ or without ${ }^{17}$ conjunctival abnormalities, and had large variations of the biopsy size. "Our biopsy size was consistent from patient to patient and larger than in many of the studies. In past studies the range of positive conjunctival biopsies ranged from 0 to $75 \%$, with a weighted average of $24 \%$. In our study conjunctival biopsies were positive in $66.7 \%$ with and $31.4 \%$ without conjunctival follicles. Thus we found blind biopsies can be useful in the diagnosis of sarcoidosis, which is in contrast to previous studies that have reported blind conjunctival biopsies being valueless. ${ }^{14} 19$

Although our study did not have any control patients, three previous studies have addressed the incidence of false positive conjunctival biopsies. ${ }^{11214}$ The total number of control conjunctival biopsies in these three studies was 116 , and many of these patients had other granulomatous disorders. There were no false positives. In our study and in the previous studies, ${ }^{10-}$ ${ }^{17}$ no serious complications have been reported from conjunctival biopsy.

Angiotensin converting enzyme has been used to help make the diagnosis of sarcoidosis, but there are two problems with using the ACE test. The first is its low sensitivity. The second is that every disease that would be included in a differential diagnosis with sarcoidosis can also cause a rise in ACE levels. ${ }^{2324}$ Although the histological picture of sarcoidosis - non-caseating granulomas - is not pathognomonic, histopathological examination of tissue provides information that helps to discriminate between the elements in the differential diagnosis of sarcoidosis, something the ACE test does not do efficiently.

We found biopsy of the conjunctiva to be an effective and useful method of obtaining this histopathological information, and it seemed particularly worthwhile in patients with advanced chest $x$ ray stages or ocular abnormalities. Transbronchial lung biopsy is expensive and invasive, and carries a greater risk of serious complication than conjunctival biopsy. A practical biopsy strategy would be to biopsy the conjunctiva of sarcoidosis suspects and, only if the conjunctival biopsy is negative, do a transbronchial lung biopsy. Limiting the conjunctival biopsy to those patients with ocular abnormalities or advanced chest $x$ ray stages might prove to be even more cost effective.

The members of the Pulmonary Service, Dr David Russell, Dr David Goya, and Dr Freddie Negron performed the transbronchial biopsies on the patients in this study.

This work was supported in part by LuEsther T Mertz Retinal Research Fund, Manhattan Eye, Ear and Throat Hospital, New York.

1 James DG, Turiaf J, Hosada $\mathrm{Y}$, et al. Description of sarcoidosis: report of the subcommittee on classification and definition. Ann NY Acad Sci 1976; 278: 742.

2 Jabs DA, Johns CJ. Ocular involvement in chronic sarcoidosis. Am f Ophthalmol 1986; 102: 297-301.

3 Obenauf CD, Shaw HE, Sydnor CF, Klintworth GK. Sarcoidosis and its ophthalmic manifestations. $A m \dot{f}$ Ophthalmol 1978; 86: 648-55.

4 Iwata K, Nanba K, Sobue K, Abe H. Ocular sarcoidosis: evaluation of intraocular findings. Ann NY Acad Sci 1976; 278: $445-54$.

5 James DG. Ocular sarcoidosis. Ann NY Acad Sci 1986; 465; $551-63$.

6 Crick RP, Hoyle C, Smellie H. The eyes in sarcoidosis. $\mathrm{Br} F$ Ophthalmol 1961; 45: 461-81.

7 Dresner MS, Brecher R, Henkind P. Ophthalmology consultation in the diagnosis and treatment of sarcoidosis. Arch Intern Med 1986; 146: 301-4.

8 Daniel TM. Sarcoidosis. In: Baum GL, Wolinsky E, eds. Textbook of pulmonary diseases. Boston: Little Brown, 1983: 685-701.

9 Daniele RP. Sarcoidosis: diagnosis and management. Hosp $\operatorname{Prac}($ Off) 1983; 18: 113-22.

10 Karcioglu ZA, Brear R. Conjunctival biopsy in sarcoidosis. Am f Ophthalmol 1985; 99: 68-73.

11 Khan F, Wessely Z, Chazin SR, Seriff NS. Conjunctival biopsy in sarcoidosis: a simple, safe, and specific diagnostic procedure. Ann Ophthalmol 1977; 9: 671-6.

12 Bornstein JS, Frank MI, Radner DB. Conjunctival biopsy in the diagnosis of sarcoidosis. N Engl f Med 1962; 267: 60-4.

13 Solomon DA, Horn BR, Byrd RB, Lorfel RS, Griggs GA. The diagnosis of sarcoidosis by conjunctival biopsy. Chest 1978; 74: 271-3.

14 Crick R, Hoyle C, Mather G. Conjunctival biopsy in sarcoidosis. BrMed F 1961; ii: 1180-1.

15 Karma A, Sutinen S, Karma P. Conjunctival and tonsillar biopsies in sarcoidosis. Chest 1980; 78: 900-1.

16 Merritt JC, Lipper SL, Peiffer RL, Hale LM. Conjunctival biopsy in sarcoidosis. 7 Natl Med Assoc 1980; 72: 347-9.

17 Nichols CW, Eagle RC, Yanoff M, Menocal NG. Conjunctival biopsy as an aid in the evaluation of the patient with sarcoidosis. Ophthalmology 1980; 87: 287-91.

18 DeRemee RA. The roentgenographic staging of sarcoidosis. Historic and contemporary perspectives. Chest 1983; 83: 128-33.

19 James DG. Ocular sarcoidosis. Am f Med 1959; 26: 331-9.

20 Gronhagen-Riska C, Serlos O, Wagar G, Fyhrquisf. Angiotensin converting enzyme. II. Serum activity in early and newly diagnosed sarcoidosis. Scand $\mathcal{F}$ Resp Dis 1979; 60: 94 101

21 Romer FK, Angiotensin-converting enzyme in newly detected sarcoidosis: with special reference to enzyme levels in sarcoidosis: with special reference to enzyme levels in patients with

22 Rizzato G, Blasi A. A European survey on the usefulness of ${ }^{67} \mathrm{GA}$ lung scans in assessing sarcoidosis. Experience in 14 research centers in seven different counties. Ann NY Acad Sci $1986 ; 465: 463-78$

23 Studdy PR, Lapworth R, Bird R. Angiotensin converting enzyme and its clinical significance - a review. 7 Clin Pathol 1983; 36: 938-47.

24 Gronhagen-Riska C. Angiotensin converting enzyme. I. Activity and correlation with serum lysozyme in sarcoidosis, other chest or lymph node diseases and healthy persons. Scand $\mathcal{F}$ Resp Dis 1979; 60: 83-93. 